\title{
Alternating electric tumor treating fields for treatment of glioblastoma: rationale, preclinical, and clinical studies
}

\author{
Sandeep Mittal, MD, FRCSC,1,2,5 Neil V. Klinger, MS, ${ }^{1}$ Sharon K. Michelhaugh, PhD, ${ }^{1}$ \\ Geoffrey R. Barger, MD, ${ }^{3,5}$ Susan C. Pannullo, MD, ${ }^{6,7}$ and Csaba Juhász, MD, PhD ${ }^{3-5}$
}

Departments of ${ }^{1}$ Neurosurgery, ${ }^{2}$ Oncology, ${ }^{3}$ Neurology, and ${ }^{4}$ Pediatrics, ${ }^{5}$ Karmanos Cancer Institute, Wayne State University, Detroit, Michigan; ${ }^{6}$ Department of Neurological Surgery, NewYork-Presbyterian Hospital/Weill Cornell Medical Center, New York; and 'Department of Biomedical Engineering, Cornell University, Ithaca, New York

OBJECTIVE Treatment for glioblastoma (GBM) remains largely unsuccessful, even with aggressive combined treatment via surgery, radiotherapy, and chemotherapy. Tumor treating fields (TTFs) are low-intensity, intermediate-frequency, alternating electric fields that have antiproliferative properties in vitro and in vivo. The authors provide an up-to-date review of the mechanism of action as well as preclinical and clinical data on TTFs.

METHODS A systematic review of the literature was performed using the terms "tumor treating fields," "alternating electric fields," "glioblastoma," "Optune," "NovoTTF-100A," and "Novocure."

RESULTS Preclinical and clinical data have demonstrated the potential efficacy of TTFs for treatment of GBM, leading to several pilot studies, clinical trials, and, in 2011, FDA approval for its use as salvage therapy for recurrent GBM and, in 2015, approval for newly diagnosed GBM.

CONCLUSIONS Current evidence supports the use of TTFs as an efficacious, antimitotic treatment with minimal toxicity in patients with newly diagnosed and recurrent GBM. Additional studies are needed to further optimize patient selection, determine cost-effectiveness, and assess the full impact on quality of life.

https://thejns.org/doi/abs/10.3171/2016.9.JNS16452

KEY WORDS alternating electric fields; newly diagnosed glioblastoma; recurrent glioblastoma; Optune; NovoTTF-100A; tumor treating fields; oncology

$\mathrm{G}$ LIOBLASTOMA (GBM) is the most common malignant primary CNS tumor. Despite advances with the combined therapy of surgery, radiation, and chemotherapies such as temozolomide (TMZ; alkylating agent, interfering with DNA replication) and bevacizumab (recombinant antibody to vascular endothelial growth factor A [VEGF-A], inhibiting angiogenesis), median survival is still dismal at $14-16$ months. ${ }^{8,22,28}$ In addition, these treatments are typically toxic to the patient, despite improving survival. New therapeutic approaches are needed to improve the outcomes and quality of life (QOL) of GBM patients. Tumor treating fields (TTFs) are low-intensity, intermediate-frequency, alternating electric fields that have demonstrable antiproliferative properties in vitro and in vivo. ${ }^{710}$ We searched the literature and here provide an up-to-date review of the mechanism of action as well as the preclinical and clinical data on TTFs.

\section{Methods}

A literature search was performed using the MEDLINE database, as was a manual search of bibliographies of the resultant articles. The following search terms were used: "tumor treating fields," "alternating electric fields," "glioblastoma," "Optune," "NovoTTF-100A," and "Novocure." Articles were identified independently and full text content was reviewed.

\section{Results}

The successful application of this technology has been

ABBREVIATIONS GBM = glioblastoma; $L Y G=$ life years gained; $M D R=$ multidrug resistant; $N S C L C=$ non-small cell lung cancer; OS = overall survival; $P F S=$ progression-free survival; $\mathrm{QALY}=$ quality-adjusted life year; $\mathrm{QOL}$ = quality of life; TMZ = temozolomide; TTF = tumor treating field; TTP = time to disease progression. SUBMITTED February 24, 2016. ACCEPTED September 22, 2016.

INCLUDE WHEN CITING Published online February 24, 2017; DOI: 10.3171/2016.9.JNS16452. 
observed in several pilot studies and clinical trials and prompted FDA approval in April 2011 for use as a salvage monotherapy for GBM patients (see approved indications and contraindications in Table 1). ${ }^{10,13,17,19,25}$ In October 2015, Optune (formerly known as NovoTTF-100A, Novocure Ltd.) was approved for use with TMZ for the treatment of adults with newly diagnosed supratentorial GBM following maximal surgical debulking and completion of radiation therapy with concomitant standard-of-care chemotherapy. ${ }^{27}$

Several clinical trials are evaluating TTFs for use in GBM, WHO Grade II and III meningioma, pancreatic cancer, ovarian cancer, and non-small cell lung cancer (NSCLC) brain metastases. Two phase III randomized clinical trials have been completed for newly diagnosed and recurrent GBM (Table 2).

\section{TTFs: Rationale}

Despite aggressive treatment, outcomes for GBM patients remain bleak. A major limitation of most cancer treatments is their poor therapeutic index. ${ }^{10}$ Glioblastoma is an attractive candidate for TTFs because although this lesion type diffusely infiltrates the brain, ${ }^{28}$ it almost never metastasizes, ${ }^{25}$ making GBM particularly suitable for regional therapy such as alternating electric fields. Since TTFs exert their effects via physical rather than biological or chemical mechanisms, the therapeutic effect is less likely to be dependent on one specific cellular characteristic such as cell surface receptor expression. As such, TTFs are expected to be an efficacious therapy against many tumor types, as well as less sensitive to mutation immunity, similar to radiation therapy. ${ }^{10}$ Unlike with radiation, however, there is a much lower risk of toxicity to the patient. In addition, TTFs inherently avoid the issue of delivering treatment across the blood-brain barrier, a major experimental and clinical hurdle for cytotoxic and targeted therapies.

The current standard of care for GBM includes chemotherapy as salvage therapy. While it may improve survival in some individuals, there are chemotherapy-related toxicities that diminish patient QOL. ${ }^{25}$ In GBM patients, TTFs as monotherapy have been shown to perform as well as chemotherapy, without harmful side effects.

\section{TTFs: Mechanism of Action}

Cells contain ions and polar molecules that are responsive to and generate electric fields. ${ }^{10}$ Ions and polar molecules behave differently when placed within electric fields. Ions tend to flow in a field, whereas polar molecules orient themselves along the field lines. ${ }^{9110}$ However, this occurs only in uniform electric fields. Nonuniform electric fields cause polar molecules to move toward higher field intensities, a process is known as "dielectrophoresis."

Alternating electric fields with intermediate frequencies (range $100 \mathrm{kHz}$ to $1 \mathrm{MHz}$ ) have significant effects on cells undergoing mitosis, driving these dividing cells to apoptosis, while having no major effects on nondividing cells. ${ }^{12}$ This interference with replication has been the basis for TTFs in cancer therapy, since tumor cells replicate much faster than most normal cells. ${ }^{10}$ Time-lapse micro-
TABLE 1. Current FDA-approved indications and contraindications of Optune

\begin{tabular}{l}
\hline Recurrent GBM (FDA approval on April 8, 2011) \\
Indications \\
Age 22 yrs or older \\
Confirmed recurrent GBM after chemotherapy \\
GBM in supratentorial location \\
To be used as monotherapy \\
As alternative to standard medical therapy after surgical \& radiation \\
options exhausted \\
Contraindications \\
Active implanted medical device present* \\
Skull defect present \\
Known sensitivity to conductive hydrogels \\
\hline Newly Diagnosed GBM (FDA approval on October 5, 2015) \\
\hline Indications \\
Age 22 yrs or older, GBM in supratentorial location \\
Confirmed newly diagnosed GBM following maximal debulking sur- \\
gery \& completion of radiation therapy w/ concomitant standard- \\
of-care chemotherapy \\
To be used w/ TMZ \\
\hline Contraindications \\
Active implanted medical device present* \\
Skull defect present \\
Known sensitivity to conductive hydrogels \\
\hline * Devices include deep brain stimulators, spinal cord stimulators, vagus nerve \\
stimulators, pacemakers, defibrillators, programmable shunts.
\end{tabular}

photography has shown that mitosis is prolonged in dividing cells exposed to TTFs. ${ }^{12}$ In addition, cells undergoing mitosis while being exposed to TTFs may be destroyed as the formation of the cleavage furrow proceeds and the cell membrane ruptures. Two mechanisms are thought to play a role in cellular death due to TTFs: microtubule subunit misalignment and dielectrophoretic movement of organelles and macromolecules..$^{10,12,25}$ Failure of cytokinetic furrow formation can lead to inappropriate chromosome segregation and eventual cell death. ${ }^{7}$ During cytokinesis, furrow formation is expected to cause distortion in the applied electric fields to become nonuniform (heterogeneous; Fig. 1). This induces unidirectional forces (dielectrophoresis) that interfere with spindle tubulin. ${ }^{6,10}$ These effects are similar to those caused by drugs (for example, paclitaxel or vinca alkaloid derivatives) that directly interfere with microtubule polymerization. ${ }^{18,19}$ The induced forces depend on the angle between the field and the axis of division. Cells aligned with applied fields are more strongly affected than those that are unaligned. Applying several fields sequentially in multiple directions allows the TTFs therapy to overcome this natural barrier and increase its antiproliferative effectiveness in vitro and in vivo. ${ }^{10}$ It has been shown that the antiproliferative effects are dependent on the mitotic spindle orientation in relation to the field. ${ }^{19}$

The Optune device delivers intermediate-frequency (100-200 kHz), low-intensity (1-2 V/cm), alternating electric fields through disposable ceramic transducer arrays placed on the patient's shaved scalp. ${ }^{19}$ The device is battery operated to increase portability and allow for patient mobility during use and is preset by treatment personnel, 
TABLE 2. Summary of clinical trials using Optune (NovoTTF-100A)

\begin{tabular}{|c|c|c|c|c|c|}
\hline $\begin{array}{l}\text { Trial } \\
\text { Registration } \\
\text { No. }\end{array}$ & $\begin{array}{l}\text { Study } \\
\text { Name }\end{array}$ & $\begin{array}{l}\text { Outcome } \\
\text { Measures }\end{array}$ & Phase & $\begin{array}{l}\text { Start } \\
\text { Date }\end{array}$ & $\begin{array}{l}\text { Primary } \\
\text { Completion } \\
\text { Date }\end{array}$ \\
\hline \multicolumn{6}{|c|}{ Completed studies } \\
\hline NCT00379470 & $\begin{array}{l}\text { Effect of NovoTTF-100A in Recur- } \\
\text { rent Glioblastoma Multiforme } \\
\text { (GBM) }\end{array}$ & $\begin{array}{l}\text { OS, PFS at } 6 \text { mos, median TTP, \% 1-year survival, radiologi- } \\
\text { cal response (Macdonald criteria), QOL assessment } \\
\text { (EORTC QLQ-C30), adverse events severity \& frequency }\end{array}$ & III & $09 / 2006$ & $11 / 2009$ \\
\hline NCT00916409 & $\begin{array}{l}\text { Effect of NovoTTF-100A Together } \\
\text { With Temozolomide in Newly } \\
\text { Diagnosed Glioblastoma Multi- } \\
\text { forme (GBM) }\end{array}$ & PFS time, OS & III & $06 / 2009$ & $\begin{array}{l}\text { 11/2014 } \\
\text { (study } \\
\text { terminated } \\
\text { early) }\end{array}$ \\
\hline \multicolumn{6}{|l|}{ Active studies } \\
\hline NCT01755624 & $\begin{array}{l}\text { Effect of TTFields (150kHz) in } \\
\text { Non-small Cell Lung Cancer } \\
\text { (NSCLC) Patients With } 1-5 \\
\text { Brain Metastases Following Op- } \\
\text { timal Standard Local Treatment }\end{array}$ & $\begin{array}{l}\text { Time to local \& distant progression in brain, OS, 6-mo dis- } \\
\text { ease control rate in brain, neurocognitive function (MMSE } \\
\text { \& HVLT), QOL (QLQ-C30 and QLQ-BN20), overall } \\
\text { (systemic) PFS (RECIST Criteria), adverse events severity } \\
\text { \& frequency }\end{array}$ & II & $01 / 2013$ & $12 / 2014$ \\
\hline NCT01756729 & $\begin{array}{l}\text { Post-approval Study of NovoTTF- } \\
100 \mathrm{~A} \text { in Recurrent GBM } \\
\text { Patients }\end{array}$ & $\begin{array}{l}\text { OS, change in neurocognitive function from baseline based } \\
\text { on MMSE, genetic profiling of tumors \& correlation w/ } \\
\text { response to NovoTTF-100A treatment, adverse events } \\
\text { severity \& frequency }\end{array}$ & IV & $12 / 2012$ & $01 / 2018$ \\
\hline NCT01892397 & $\begin{array}{l}\text { Pilot Study of Optune (NovoTTF- } \\
\text { 100A) for Recurrent Atypical } \\
\text { and Anaplastic Meningioma }\end{array}$ & PFS rate, OS, safety \& tolerability & & $06 / 2013$ & $06 / 2015$ \\
\hline NCT01894061 & $\begin{array}{l}\text { NovoTTF-100A With Bevacizu- } \\
\text { mab (Avastin) in Patients With } \\
\text { Recurrent Glioblastoma }\end{array}$ & $\begin{array}{l}\text { PFS, objective response rate based on RANO Criteria, no. of } \\
\text { patients who experience toxicities w/ this combination of } \\
\text { therapies, median OS, TTP, neurocognitive function, QOL }\end{array}$ & II & $06 / 2013$ & $12 / 2014$ \\
\hline NCT01925573 & $\begin{array}{l}\text { Optune (NOVOTTF-100A) + } \\
\text { Bevacizumab + Hypofraction- } \\
\text { ated Stereotactic Irradiation } \\
\text { Bevacizumab-Naive Recurrent } \\
\text { Glioblastoma (GCC 1344) }\end{array}$ & $\begin{array}{l}\text { No. of adverse events w/ Grade } 3 \text { or high toxicity (primary } \\
\text { measure) }\end{array}$ & & $15 / 2014$ & $12 / 2016$ \\
\hline NCT01954576 & $\begin{array}{l}\text { NovoTTF Therapy in Treating } \\
\text { Patients With Recurrent Glio- } \\
\text { blastoma Multiforme }\end{array}$ & $\begin{array}{l}\text { ORR (CR + PR + SD) (bevacizumab-naive), ORR (CR + } \\
\text { PR + SD) (bevacizumab-refractory), genetic signature of } \\
\text { response (CR + PR + SD), genetic signature of response } \\
\text { (CR + PR) \& SD, PFS in bevacizumab-naive \& bevaci- } \\
\text { zumab-refractory glioblastoma patients, QOL }\end{array}$ & II & 10/2013 & $05 / 2016$ \\
\hline
\end{tabular}

$\mathrm{CR}=$ complete response; EORTC = European Organisation for Research and Treatment of Cancer; HVLT = Hopkins Verbal Learning Test; MMSE = Mini-Mental State Examination; $\mathrm{ORR}=$ objective response rate; $\mathrm{PR}=$ partial response; $\mathrm{RANO}=$ Response Assessment in Neuro-Oncology; $\mathrm{RECIST}=$ Response Evaluation Criteria in Solid Tumors; SD = stable disease.

thus not requiring the patient to control it (Fig. 2). ${ }^{17,19}$ The smaller and lighter second-generation Optune system was approved by the FDA on July 13, 2016.

\section{Preclinical Studies}

Initial Studies With Various Tumor Cell Lines In Vitro and In Vivo

The first study to show that low-intensity, intermediatefrequency, alternating electric fields inhibit cell division was published in 2004. ${ }^{12}$ Authors of this study examined the effects of these fields on 11 in vitro cancer cell lines, including human malignant glioma, lung cancer, breast cancer, prostate cancer, melanoma, as well as mouse melanoma and adenocarcinoma and rat malignant glioma cell lines. In all 11 cell lines tested, 24-hour exposure at field intensities of $1.0-1.4 \mathrm{~V} / \mathrm{cm}$ and $100 \mathrm{kHz}$ caused significant mitotic inhibition, which was observed up to 72 hours posttreatment. ${ }^{12}$ Noncancerous baby hamster kidney cells exposed to TTFs were unaffected, as were various nonreplicating rat tissues, further demonstrating that TTFs only affect tissues that are undergoing rapid cellular division. ${ }^{12}$ Kirson et al. demonstrated that there is an optimal frequency for maximum inhibition of different tumor cell types, where the optimal TTFs frequency is inversely related to cell size.$^{10}$ Optimum frequency $(200 \mathrm{kHz})$ was observed across the malignant glioma cell lines tested.

In in vivo studies of tumor-bearing mice, in which the animals were subsequently exposed to TTFs for 3-6 days $(100-200 \mathrm{kHz},<2 \mathrm{~V} / \mathrm{cm})$, the TTFs were effective in 

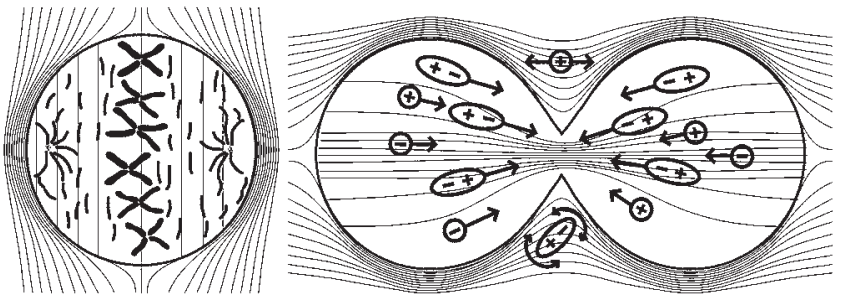

FIG. 1. Mechanism of action of alternating electric fields on intracellular structures during mitosis. During metaphase (left), the electric field is uniform within the cell, causing tubulin subunits to align with the direction of the field and inhibiting their polymerization and hence interfering with the formation of a normal microtubule spindle. During cytokinesis (right), the electric field is nonuniform within the dividing cell and drives charged and polar macromolecules and organelles toward the high field density at the mitotic furrow. This process interferes with spindle tubulin orientation and induces dielectrophoresis, thereby disrupting mitosis and disintegrating the daughter cells. Copyright Novocure, Inc. Published with permission.

inhibiting tumor growth. When multiple TTFs were applied sequentially in different directions, the diameter of the treated tumor was about half the size of the untreated control. ${ }^{10}$ Interestingly, the presence of abnormal mitotic figures was demonstrated in a rat glioma model following TTFs application. ${ }^{?}$

\section{Safety Studies}

Potential toxicity to human cells from the use of TTFs was addressed and subsequently ruled out because of the low field intensity. To prevent prolonged exposure of human skin to the externally applied ceramic arrays, the arrays are insulated, which lowers impedance and allows safe operating voltages to be used to generate the TTFs (http://www.accessdata.fda.gov/cdrh_docs/pdf10/ P100034c.pdf). ${ }^{16}$

\section{TTFs Combined With Chemotherapy}

The combination of TTFs and conventional chemotherapies (paclitaxel, doxorubicin, cyclophosphamide) was tested, and dose-response curves were established..$^{13}$ As the concentration of the drug increased, cell proliferation decreased. Combining TTFs with chemotherapy shifted the dose-response curves to the left, indicating that lower doses of the chemotherapy agents achieved the same growth inhibition in the presence of TTFs. ${ }^{13}$
To test TTFs with chemotherapeutic agents in malignant gliomas, cells were cultured in TTFs alone and in combination with dacarbazine. Application of the TTFs augmented the efficacy of the alkylating drug. In tumorbearing rabbits, paclitaxel alone inhibited tumor growth by $15 \%$, TTFs alone by $53 \%$, and TTFs with paclitaxel by $69 \%$. The TTFs-mediated enhanced sensitivity to chemotherapy was statistically significant in vivo. ${ }^{13}$

An in vitro study examined the potential of TTFs to treat multidrug-resistant (MDR) tumor cells. ${ }^{20}$ Both MDR cell lines and their wild-type counterparts were subjected to TTFs for 72 hours with and without chemotherapeutic agents. When MDR cells were treated with TTFs alone, a reduction in viable cells was observed; however, when TTFs were administered with chemotherapy, the viable cells decreased significantly versus chemotherapy alone. ${ }^{20}$ The TTFs augmented the cells' sensitivity to chemotherapy, not by altering drug transport but possibly by the disruptive forces of TTFs to cytoskeletal, microtubule, and mitochondrial molecules.

\section{Clinical Studies in GBM}

A small pilot study of TTFs in humans (6 subjects) demonstrated feasibility. ${ }^{19}$ Subjects (median age 66 years) had a variety of primary tumors, had been heavily pretreated for progressive disease, and had exhausted standard-of-care treatments. Selection criteria for the study included at least 1 measurable lesion, a tumor accessible by TTFs, and no concomitant antitumor therapy. Compliance in this study, $>80 \%$, demonstrated that TTFs (13-46 days) were well tolerated with no serious adverse events. Adverse events that did occur were mild (Grade 1) skin irritation beneath the electrodes and were reversible with topical steroids and electrode repositioning. Overall, 1 patient had a partial response, whereas 3 had tumor growth arrest during treatment.

The first demonstration of the efficacy of TTFs treatment was documented in 10 recurrent GBM patients..$^{10} \mathrm{~A}$ TTFs treatment of 280 weeks yielded no serious adverse events or changes in serum chemistry and blood count in any patient. Most of the patients had mild to moderate contact dermatitis in the treatment area, which responded to topical steroids and electrode relocation. These reactions were probably caused by chronic moisture, heat, or chemical irritation by the hydrogel and medical tape. ${ }^{1}$
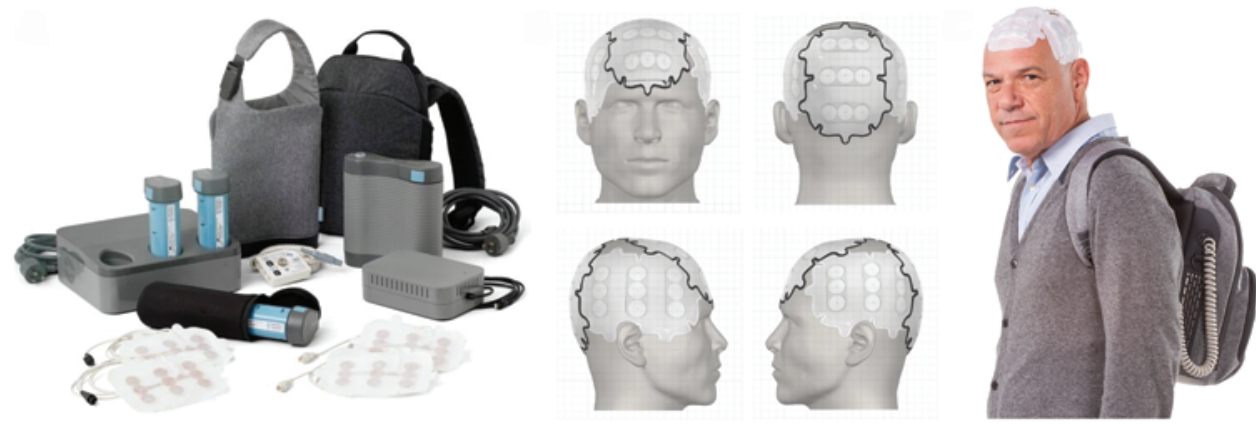

FIG. 2. The Optune system for treatment of recurrent GBM in adults was approved by the FDA in April 2011. Patients are required to use the system for at least 18 hours daily. The system contains several transducer arrays (electrodes) that are applied directly to the shaved scalp and a battery pack that can be carried in a bag. Copyright Novocure, Inc. Published with permission. 


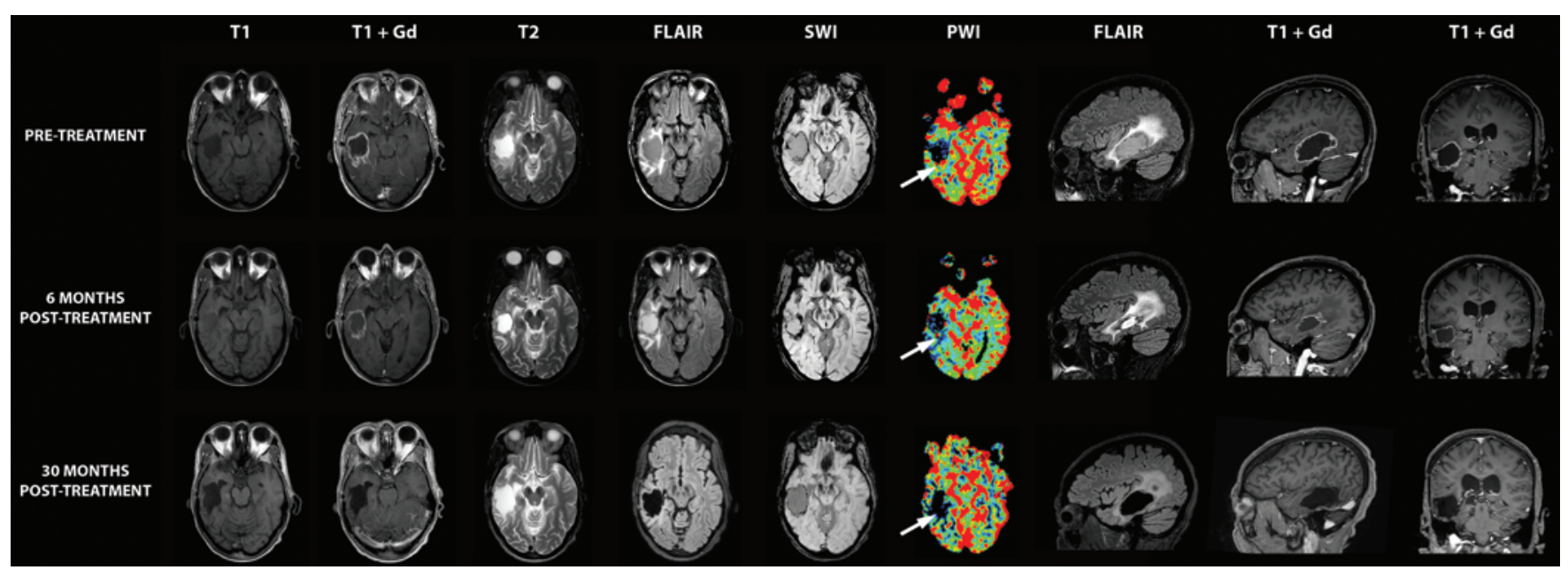

FIG. 3. Case illustration. A 55-year-old woman underwent resection of a right temporal GBM followed by adjuvant radiation therapy and chemotherapy (standard regimen in Stupp et al., 2012). Follow-up brain MRI after 5 cycles of TMZ showed evidence of recurrent tumor (pretreatment, upper). Repeat surgery was recommended; however, the patient refused and was very motivated to initiate NovoTTF therapy. After 6 months of continuous treatment (compliance $>80 \%$ ), the patient remained neurologically stable with an improved radiological appearance of the right temporal GBM (6 months posttreatment, middle). Noncontrast and postcontrast T1-weighted MR images show decrease in size and contrast enhancement following NovoTTF treatment. The patient has remained neurologically stable after 2.5 years of therapy (30 months posttreatment, lower) with no radiological evidence of tumor recurrence. Persistent peritumoral hyperintense signal changes on T2 and FLAIR images were noted. Increased susceptibility artifact was noted along the enhancing portion of the tumor after NovoTTF therapy on susceptibility-weighted images, which has resolved on long-term follow-up. An area of increased cerebral blood volume was noted along the posterior enhancing margin of the tumor on perfusion-weighted imaging (white arrow, upper). Interestingly, after 6 months of NovoTTF therapy, perfusion analysis demonstrated an overall decreased relative cerebral blood volume in the region of the mass and surrounding parenchyma (white arrow, middle). Low perfusion remained in this area on long-term follow-up (white arrow, lower). PWI = perfusion-weighted imaging; SWI = susceptibility-weighted imaging.

Patients were treated for 18 hours/day until tumor progression was evident on neuroimaging. ${ }^{10}$ This length of time was determined based on in vitro data showing that TTFs are most effective when applied continuously for at least 16 hours. In that study, the median time to disease progression (TTP) was 26.1 weeks and progression-free survival (PFS) at 6 months was 50\%. The median overall survival (OS) for these 10 recurrent GBM patients was 62.2 weeks. The OS and TTP values obtained with TTFs were more than twice the median historical control values at that time..$^{10}$

Given the results of the GBM pilot study, an international, multi-institutional phase III trial of recurrent GBM treatment was launched..$^{25}$ In this study, Optune was tested as monotherapy and compared with an active chemotherapy control group. The primary end point of this study was an increase in OS. Inclusion criteria included an age $\geq 18$ years, prior radiotherapy, histologically confirmed recurrent GBM, and radiologically confirmed disease progression with no limit on previous therapies or recurrences. Patients with infratentorial tumors or with implanted medical devices were excluded from the study. The patient population (237 patients, median age 54 years) was divided between monotherapy (120 patients) and control (117 patients) groups, and $>80 \%$ of the entire study cohort had failed 2 or more prior chemotherapies. Data from a log file on the device indicated that compliance was $86 \%$ (20.6 hours/day). In the active chemotherapy control group, patients were mainly treated with bevacizumab or irinotecan (31\% each), nitrosourea (25\%), carboplatin (13\%), or
TMZ (11\%). Median survival was 6.6 months in the TTFs group, compared with 6.0 months in controls. Two- and 3 -year survival rates were $8 \%$ and $4 \%$, respectively, in the TTFs group, compared with $5 \%$ and $1 \%$ in controls. The hazard ratio $(0.86,95 \%$ CI $0.66-1.12)$ indicated that TTFs monotherapy was at least as effective as chemotherapy. No systemic side effects were observed in the TTFs group, with only Grade 1 or 2 contact dermatitis beneath the transducer arrays in $16 \%$ of the patients. Longitudinal QOL data were analyzed in patients when available (63 patients [27\%]). Improvements in QOL were reported for cognitive, emotional, and role functioning in the TTFs patients, with a potential decrease in physical functioning. Symptoms related to chemotherapy included appetite loss, diarrhea, constipation, nausea, vomiting, pain, and fatigue. Given the progressive nature of GBM, 220 patients had died at a median follow-up of 39 months. Patients received supportive care after tumor progression, with only 5.8\% of TTFs patients and $10.3 \%$ of controls receiving further salvage therapy.

Although the primary end point (increased OS as compared with survival in chemotherapy controls) was not met in this study, the TTFs therapy was effective as a monotherapy with the added benefits of avoiding toxicity and improving QOL. ${ }^{25}$ More patients showed objective radiological responses in the TTFs group than in the active chemotherapy control group, although the difference was not significant because of the relatively low numbers of patients (see our illustrative case in Fig. 3). 


\section{TTFs for Newly Diagnosed GBM}

A single-center pilot clinical study was performed in 10 subjects with newly diagnosed GBM ${ }^{13}$ In this trial, median PFS for subjects treated with the TTFs device was 14.4 months, which was better than PFS for historical controls (7.1 months). ${ }^{13}$ The study concluded after 4 years, when 3 patients were alive with no tumor progression, 5 had died, and 2 were lost to follow-up. Median OS from the time of initial diagnosis exceeded 40 months, as compared with 14.7 months of historical controls. Mild to moderate scalp irritation attributed to the transducer arrays was the only adverse event that could be directly linked to the TTFs device. Findings from this pilot study, along with preliminary results of the EF-11 study, prompted a prospective, randomized, open-label controlled trial examining the safety and efficacy of TTFs in combination with TMZ compared with TMZ alone in subjects with newly diagnosed GBM.

\section{EF-14 Data}

To further establish that TTFs treatment is a valuable addition to current standard of care for newly diagnosed GBM, a clinical trial (NCT00916409) was performed..$^{23,24}$ The study was terminated early after finding significant increases in PFS and OS in patients treated using TTFs with TMZ. Patients treated with Optune and TMZ (210 patients) had statistically significant improvements in median PFS (7.1 vs 4.0 months, $\mathrm{p}=0.001$ ) and OS (19.6 vs 16.6 months, $\mathrm{p}=0.034)$ compared with patients treated with TMZ alone (105 patients). ${ }^{24}$ These data support the use of TTFs combined with TMZ in the treatment of newly diagnosed GBM. ${ }^{23}$

In this pivotal EF-14 trial, 695 patients were randomized 2:1 to undergo treatment with TTFs along with maintenance TMZ following chemoradiation for histologically proven GBM. An interim analysis on the first 315 randomized subjects and a final analysis with the intent-totreat study population data on PFS and OS were recently published..$^{23,24}$ The PFS (primary end point) benefit at the interim and final analysis was 3 months, which was statistically significant. The OS benefit (secondary end point) was also statistically significant at 3 months in the interim analysis (19.6 vs 16.6 months) and final analysis (19.4 vs 16.6 months) in subjects randomized to TTFs plus TMZ versus TMZ alone. Quality of life assessments (including metrics for cognitive function and functional status) demonstrated no detriment related to device use. Adverse events attributed to the TTFs included skin irritation, although Grade 3 skin toxicity occurred in only $2 \%$ of those treated with TTFs plus TMZ. ${ }^{23}$

Long-term analysis of the full data set of 695 patients enrolled in the trial confirmed the findings of the interim analysis. The addition of TTFs to TMZ improved PFS by 2.7 months (from 4 to 6.7 months) and OS by 4.8 months (from 16 to 20.8 months from randomization, or from 19.8 to 24.5 months from diagnosis). ${ }^{21}$

Overall, the EF-14 data demonstrated prolongation of both PFS and OS with the use of TTFs administered concurrently with maintenance TMZ, after surgery plus postoperative chemoradiation, as compared with maintenance TMZ alone, and with minimal skin toxicity and no detrimental effect on QOL measures. ${ }^{23}$

\section{Ongoing Clinical Trials}

Because TTFs show promise in treating GBM patients, more clinical trials have been launched to further explore their potential. Currently, 6 neurooncology clinical trials of TTFs are actively recruiting patients (Table 2). Four clinical trials are focused on treating GBM, 1 on atypical and anaplastic meningioma, and 1 on NSCLC brain metastases. For GBM, studies of TTFs in combination with bevacizumab or with radiotherapy and bevacizumab are underway. A unique phase II pilot study (NCT01954576) that began in October 2013 is examining TTFs in recurrent GBM patients while performing genetic biomarker analysis to identify the genetic signature of recurrent GBM as it responds to treatment. The pilot study treating high-grade meningiomas, which have a high rate of recurrence, short survival time, and limited treatment options, ${ }^{4}$ is expected to be completed in mid-2017. Non-small cell lung cancer brain metastases are also being studied. Since TTFs are efficacious in primary lung tumors (NSCLC) ${ }^{17}$ and in intracranial tumors (GBM), ${ }^{10,19,25}$ TTFs may also be effective in treating NSCLC metastases to the brain, especially given that preclinical data support this hypothesis. ${ }^{11}$

\section{Discussion}

\section{Transducer Array Layout}

Tumor treating fields customize treatment through the creation of individualized "treatment maps." Array application is adapted to a specific region as defined by brain imaging provided by the treating physician. Novocure's proprietary transducer array layout (NovoTAL) system is used to maximize field intensity to the target site of disease. Current data suggest that at recurrence, patients should have their tumors remapped and the TTF measurements adjusted. ${ }^{23}$ Multifocal disease is difficult to treat with TTFs since optimizing the field in one location may cause field intensity to be lowered in another location, thereby decreasing therapeutic efficacy at that site. ${ }^{26}$ Prescribing the device requires certification. Optune's instructions for use require prescribing medical personnel to complete a training course provided by Novocure ${ }^{16}$ In addition, physicians can be trained and certified to use the NovoTAL system for independent TAL mapping on their patients. ${ }^{3}$

\section{Patient Concerns}

In our experience, some patients find Optune to be cumbersome in its current design; however, future design changes may improve device tolerability and compliance. Clinical trials have suggested improved efficacy with at least 18 hours per day of device use, and most patients are able to comply with adequate education and support.

Patients may be reluctant to shave their heads, as is required for application of the Optune arrays. Our clinical experience echoes the findings of the 2 major randomized trials of the device, in which skin irritation was the most common side effect experienced by subjects wearing the device. This side effect is generally mild and manageable with the application of topical steroids and repositioning of the Optune arrays. ${ }^{15}$ Some patients note a sensation of heat from the arrays, but with appropriate counseling, most do not find this sensation disturbing. 


\section{Cost-Effectiveness of Optune Therapy}

The cost of the device is approximately $\$ 20,000$ per month. Although most insurance companies will pay for the device as an FDA-approved therapy for recurrent and newly diagnosed GBM, reimbursement may present a challenge. The National Comprehensive Cancer Network (NCCN) Guidelines have recently upgraded Optune from a Category 3 to a Category 2B treatment for recurrent GBM, making support of reimbursement easier for patients with recurrent disease.

Optimal timing of TTFs in patients with recurrent GBM in terms of efficacy remains unclear, although some data suggest that TTFs may be more effective prior to the use of bevacizumab. Similarly, data regarding relative costs of Optune versus salvage therapy such as bevacizumab are limited. In the setting of a recurrence, cost considerations may be balanced by the potentially fewer side effects from Optune versus systemic therapy, given the expectation that lower systemic toxicity may produce fewer health care costs by decreasing the expense of managing these toxicities. Quality-adjusted life year (QALY), an increasingly commonly used metric for the economic burden of a given therapy, has been estimated at approximately $\$ 787,000$ / QALY for bevacizumab for newly diagnosed GBM and $\$ 270,000 /$ QALY for recurrent GBM..$^{14}$ A recent French study calculated the incremental cost-effectiveness ratio (ICER) expressed as monetary costs per life-years gained (LYG) for patients with newly diagnosed GBM treated with Optune. ${ }^{2}$ In their analysis, the authors calculated that the total costs of TTF therapy and conventional therapeutic strategies were $€ 243,141$ and $€ 57,665$, respectively (incremental cost $€ 185,476$ ), resulting in an ICER of $€ 549,909$ / LYG (approximately $\$ 625,000 / \mathrm{LYG})^{2}$

\section{Conclusions}

Current evidence supports the use of TTFs as a therapeutic option for patients with GBM. The device is FDA approved for patients with both newly diagnosed and recurrent tumors. Additional studies are needed to further validate this treatment modality, optimize patient selection, determine possible benefits of synchronous or metachronous therapies, determine cost-effectiveness, and quantify impact on QOL. These studies are encouraged in the face of the few treatment options currently available for this patient population.

\section{Acknowledgments}

We acknowledge Stephen Swyberius, B.A. (Pharma Health Labs), Barbara Beiss, Ph.D. (Novocure Ltd.), and Kelly Forcucci, R.N. (Wayne State University, Department of Neurosurgery) for editorial assistance.

\section{References}

1. Ancona A, Arévalo A, Macotela E: Contact dermatitis in hospital patients. Dermatol Clin 8:95-105, 1990

2. Bernard-Arnoux F, Lamure M, Ducray F, Aulagner G, Honnorat J, Armoiry X: The cost-effectiveness of tumor-treating fields therapy in patients with newly diagnosed glioblastoma. Neuro Oncol 18:1129-1136, 2016

3. Chaudhry A, Benson L, Varshaver M, Farber O, Weinberg U,
Kirson E, et al: NovoTTF ${ }^{\mathrm{TM}}$-100A System (Tumor Treating Fields) transducer array layout planning for glioblastoma: a NovoTAL $^{\mathrm{TM}}$ system user study. World J Surg Oncol 13:316, 2015

4. Choy W, Kim W, Nagasawa D, Stramotas S, Yew A, Gopen Q, et al: The molecular genetics and tumor pathogenesis of meningiomas and the future directions of meningioma treatments. Neurosurg Focus 30(5):E6, 2011

5. Clague DS, Wheeler EK: Dielectrophoretic manipulation of macromolecules: the electric field. Phys Rev E Stat Nonlin Soft Matter Phys 64:026605, 2001

6. Gera N, Yang A, Holtzman TS, Lee SX, Wong ET, Swanson KD: Tumor treating fields perturb the localization of septins and cause aberrant mitotic exit. PLoS One 10:e0125269, 2015

7. Giladi M, Schneiderman RS, Voloshin T, Porat Y, Munster M, Blat R, et al: Mitotic spindle disruption by alternating electric fields leads to improper chromosome segregation and mitotic catastrophe in cancer cells. Sci Rep 5:18046, 2015

8. Gilbert MR, Wang M, Aldape KD, Stupp R, Hegi ME, Jaeckle KA, et al: Dose-dense temozolomide for newly diagnosed glioblastoma: a randomized phase III clinical trial. J Clin Oncol 31:4085-4091, 2013

9. Keller FJ, Gettys WE, Skove MJ: Physics, Classical and Modern. New York: McGraw-Hill, 1993, Vol 51

10. Kirson ED, Dbalý V, Tovarys F, Vymazal J, Soustiel JF, Itzhaki A, et al: Alternating electric fields arrest cell proliferation in animal tumor models and human brain tumors. Proc Natl Acad Sci U S A 104:10152-10157, 2007

11. Kirson ED, Giladi M, Gurvich Z, Itzhaki A, Mordechovich D, Schneiderman RS, et al: Alternating electric fields (TTFields) inhibit metastatic spread of solid tumors to the lungs. Clin Exp Metastasis 26:633-640, 2009

12. Kirson ED, Gurvich Z, Schneiderman R, Dekel E, Itzhaki A, Wasserman Y, et al: Disruption of cancer cell replication by alternating electric fields. Cancer Res 64:3288-3295, 2004

13. Kirson ED, Schneiderman RS, Dbalý V, Tovaryš F, Vymazal J, Itzhaki A, et al: Chemotherapeutic treatment efficacy and sensitivity are increased by adjuvant alternating electric fields (TTFields). BMC Med Phys 9:1, 2009

14. Kovic B, Xie F: Economic evaluation of bevacizumab for the first-line treatment of newly diagnosed glioblastoma multiforme. J Clin Oncol 33:2296-2302, 2015

15. Lacouture ME, Davis ME, Elzinga G, Butowski N, Tran D, Villano JL, et al: Characterization and management of dermatologic adverse events with the NovoTTF-100A System, a novel anti-mitotic electric field device for the treatment of recurrent glioblastoma. Semin Oncol 41 (Suppl 4):S1-S14, 2014

16. Novocure (USA) Inc: Instructions For Use. NovoTTF-100A System. (http://www.accessdata.fda.gov/cdrh_docs/pdf10/ P100034c.pdf) [Accessed December 1, 2016]

17. Pless M, Droege C, von Moos R, Salzberg M, Betticher D: A phase I/II trial of tumor treating fields (TTFields) therapy in combination with pemetrexed for advanced non-small cell lung cancer. Lung Cancer 81:445-450, 2013

18. Rowinsky EK, Donehower RC: Paclitaxel (taxol). N Engl J Med 332:1004-1014, 1995

19. Salzberg M, Kirson E, Palti Y, Rochlitz C: A pilot study with very low-intensity, intermediate-frequency electric fields in patients with locally advanced and/or metastatic solid tumors. Onkologie 31:362-365, 2008

20. Schneiderman RS, Shmueli E, Kirson ED, Palti Y: TTFields alone and in combination with chemotherapeutic agents effectively reduce the viability of MDR cell sub-lines that overexpress ABC transporters. BMC Cancer 10:229, 2010

21. Stupp R, Idbaih A, Steinberg DM, Read W, Toms S, Barnett G, et al: LTBK-01: Prospective multi-center phase III trial of tumor treating fields together with temozolomide compared 
with temozolomide alone in patients with newly diagnosed glioblastoma. Neuro Oncol 18 (Suppl 6):i1, 2016 (Abstract)

22. Stupp R, Mason WP, van den Bent MJ, Weller M, Fisher $\mathrm{B}$, Taphoorn MJ, et al: Radiotherapy plus concomitant and adjuvant temozolomide for glioblastoma. N Engl J Med 352:987-996, 2005

23. Stupp R, Taillibert S, Kanner AA, Kesari S, Steinberg DM, Toms SA, et al: Maintenance therapy with tumor-treating fields plus temozolomide vs temozolomide alone for glioblastoma: a randomized clinical trial. JAMA 314:2535-2543, 2015

24. Stupp R, Wong E, Scott C, Taillibert S, Kanner A, Kesari S, et al: Interim analysis of the EF-14 Trial: a prospective, multi-center trial of NovoTTF-100A together with temozolomide compared to temozolomide alone in patients with newly diagnosed GBM. Neuro Oncol 16 (Suppl 5):v167, 2014 (Abstract)

25. Stupp R, Wong ET, Kanner AA, Steinberg D, Engelhard H, Heidecke V, et al: NovoTTF-100A versus physician's choice chemotherapy in recurrent glioblastoma: a randomised phase III trial of a novel treatment modality. Eur J Cancer 48:2192-2202, 2012

26. Turner SG, Gergel T, Wu H, Lacroix M, Toms SA: The effect of field strength on glioblastoma multiforme response in patients treated with the NovoTTF ${ }^{\mathrm{TM}}$-100A system. World J Surg Oncol 12:162, 2014

27. United States Food and Drug Administration: FDA approves expanded indication for medical device to treat a form of brain cancer. FDA News Release. October 5, 2015. (http:// www.fda.gov/NewsEvents/Newsroom/PressAnnouncements/ ucm465744.htm) [Accessed December 1, 2016]
28. Xie Q, Mittal S, Berens ME: Targeting adaptive glioblastoma: an overview of proliferation and invasion. Neuro Oncol 16:1575-1584, 2014

\section{Disclosures}

Drs. Mittal and Juhász have received research support from Novocure Ltd. for non-study-related effort. Dr. Pannullo has received clinical or research support from, is a consultant for, and has received honoraria and support for $\mathrm{CME}$ courses from Novocure Ltd. Drs. Mittal and Pannullo have received support from Pharma Health Labs and Novocure Ltd. for statistical analysis for this study and/or writing or editorial assistance on this paper.

\section{Author Contributions}

Conception and design: Mittal, Acquisition of data: Mittal, Klinger. Analysis and interpretation of data: Mittal, Klinger, Pannullo, Juhász. Drafting the article: Mittal, Klinger, Michelhaugh, Pannullo. Critically revising the article: Mittal, Michelhaugh, Pannullo, Juhász. Reviewed submitted version of manuscript: all authors. Approved the final version of the manuscript on behalf of all authors: Mittal. Administrative/technical/material support: Mittal. Study supervision: Mittal.

\section{Correspondence}

Sandeep Mittal, Department of Neurosurgery, Wayne State University, 4160 John R St., Ste. 930, Detroit, MI 48201. email: smittal@med.wayne.edu. 